\title{
A generating functional approach to the sd-model with strong correlations
}

\author{
Yu.A.Izyumov, N.I.Chaschin, D.S.Alexeev \\ Institute for Metal Physics of the RAS, Ural Division \\ 620219 Ekaterinburg, Russia
}

Received July 18, 2005, in final form October 18, 2005

\begin{abstract}
A Kadanoff-Baym-type generating functional approach, earlier developed by the authors to strongly correlated systems, is applied to the sd-model with strong sd-coupling. Formalism of the Hubbard $X$-operators was used, and equation for electron Green's function was derived with functional derivatives over external fluctuating fields. Iterations in this equation generate a perturbation theory near the atomic limit. Hartree-Fock type approximation is developed within the framework of this theory, and the problem of a metal-insulator phase transition in sd-model is discussed.
\end{abstract}

Key words: theories and models of many-electron systems, lattice fermion models, strongly correlated electron systems

PACS: $71.10 .-w, 71.10 . F d, 71.27 .+a$

\section{Introduction}

In a series of papers [1-3] we suggested the generating functional approach (GFA) for the basic models of strongly correlated systems: the Hubbard model, tJ-model, periodic Anderson model. The GFA is actually a generalization of a well-known Kadanoff and Baym [4] approach, suggested for the conventional fermi-systems, to more complicated models with Hamiltonians written in terms of the spin- or the Hubbard $X$-operators. The above mentioned models are just the models for which GFA is to be effectively applied.

The idea of the method is based on introducing the generalization of the partition function $Z$ to the systems in external fields fluctuating in time and space. $Z$ is a functional of these fields. Different Green's functions (GF) of a system can be presented as functional derivatives over such fields. It is possible for each model to derive equations for basic GFs in terms of the functional derivatives. It turns out that for different models of strongly correlated systems these equations have a similar structure, which indicates the tight relations between them. It is remarkable that iterations in these equations generate a perturbation theory near the atomic limit.

The GFA and its applications to different models were discussed in detail in a 
review [5] and a monograph [6], and here we briefly reproduce the main steps of the method. The generating functional is determined by relation

$$
Z[V]=\operatorname{Tr}\left(\mathrm{e}^{-\beta \mathcal{H}} T \mathrm{e}^{-V}\right) \equiv \mathrm{e}^{\Phi[V]}
$$

where $\mathcal{H}$ is Hamiltonian of the system, $V$ is an operator of interaction with external fields, $T$ is a symbol of the ordering on the thermodynamic times $-\beta<\tau<\beta=$ $1 / k T$; trace is taken over all variables of the system. $V$-operator is a linear combination of the spin- or the $X$-operators and the coefficients in these combinations are just the fluctuating fields. It is clear that functional derivatives over these fields generate statistical averages of $T$-product of spin- or $X$-operators, which are just different GFs of the system.

In this paper we develop GFA for sd-exchange model described by Hamiltonian

$$
\mathcal{H}=\sum_{i j \sigma} t_{i j} c_{i \sigma}^{+} c_{i \sigma}-\frac{J}{2} \sum_{i}\left(\boldsymbol{S}_{i} \boldsymbol{\sigma}_{i}\right)
$$

Here the first term presents electron hopping on the lattice and the second one describes interaction of a localized spin $\boldsymbol{S}_{i}$ with electron spin $\boldsymbol{\sigma}_{i} / 2$, where $\boldsymbol{\sigma}$ is vector with Pauli matrices. Under strong sd-coupling $J \gtrsim W, W$ is a width of the band, the sd-exchange can be treated as Hamiltonian of zero approximation, and hopping as a perturbation. Practical realization of the perturbation theory over the parameter $W / J$ is based on the fact that sd-exchange Hamiltonian is the one-site one.

Eigen functions of sd-exchange Hamiltonian $-\left(\boldsymbol{S}_{i} \boldsymbol{\sigma}_{i}\right) \cdot J / 2$ are known; there are four of them [7]:

$$
\begin{aligned}
& |M 0\rangle=|M\rangle|0\rangle, \\
& |M 2\rangle=|M\rangle|2\rangle, \\
& \left|M^{+}+\right\rangle=u_{M}\left|M-\frac{1}{2}\right\rangle|\uparrow\rangle+v_{M}\left|M+\frac{1}{2}\right\rangle|\downarrow\rangle, \\
& \left|M^{-}-\right\rangle=v_{M}\left|M-\frac{1}{2}\right\rangle|\uparrow\rangle-u_{M}\left|M+\frac{1}{2}\right\rangle|\downarrow\rangle .
\end{aligned}
$$

Here $|0\rangle|, \uparrow\rangle,|\downarrow\rangle|, 2\rangle$ describe the states without an electron, having a one electron with spin $\sigma=\uparrow, \downarrow$, and with two electrons on a site, respectively; $|M\rangle$ is a wave function of an ion with spin projection $M=-S,-S+1, \ldots, S ; u_{M}$ and $v_{M}$ are Klebsh-Gordan coefficients:

$$
u_{M}^{2}=\frac{S+M+\frac{1}{2}}{2 S+1}, \quad v_{M}^{2}=\frac{S-M+\frac{1}{2}}{2 S+1}, \quad u_{M}^{2}+v_{M}^{2}=1 .
$$

The wave functions $\left|M^{+}\right\rangle$and $\left|M^{-}\right\rangle$describe a state of an ion with total spin $S+1 / 2$ and $S-1 / 2$ and its projection may be equal to

$$
\left.\begin{array}{ll}
-\left(S+\frac{1}{2}\right)<\cdots<M^{+}<\cdots<\left(S+\frac{1}{2}\right) & j=S+\frac{1}{2} \\
-\left(S-\frac{1}{2}\right)<\cdots<M^{-}<\cdots<\left(S-\frac{1}{2}\right) & j=S-\frac{1}{2}
\end{array}\right\} .
$$


In the basis of functions (1.3)-(1.6) sd-exchange Hamiltonian is diagonalized, and two eigen-energies are equal to

$$
\left.\begin{array}{ll}
E^{+}=-\frac{1}{2} J S \quad, \quad j=S+\frac{1}{2} \\
E^{-}=\frac{1}{2} J(S+1), & j=S-\frac{1}{2}
\end{array}\right\} .
$$

We will denote relations (1.5), (1.6) as

$$
\left|M^{\alpha} \alpha\right\rangle=\sum_{\sigma} \Theta^{\sigma \alpha}\left(M^{\alpha}\right)\left|M^{\alpha}-\frac{\sigma}{2}\right\rangle c_{\sigma}^{+}|0\rangle
$$

where

$$
\Theta^{\sigma+}(M)=\sqrt{\frac{S+\sigma M+\frac{1}{2}}{2 S+1}}, \quad \Theta^{\sigma-}(M)=\sigma \sqrt{\frac{S-\sigma M+\frac{1}{2}}{2 S+1}} .
$$

\section{Introducing $X$-operators}

Arbitrary one-site operator $\widehat{A}_{i}$ can be decomposed over the system of $X$-operators, determined based on the wave functions $|p\rangle$. By definitions

$$
X^{p q}=|p\rangle\langle q| .
$$

This decomposition is

$$
\widehat{A}_{i}=\sum_{p q}\langle p|\widehat{A}| q\rangle X_{i}^{p q}
$$

In the basis of functions (1.3)-(1.6) we have the following representation for electron operator $[8]$

$$
c_{i \sigma}=\sum_{M \alpha}\left[\Theta^{\sigma \alpha}\left(M+\frac{\sigma}{2}\right) X^{M 0 ;\left(M+\frac{\sigma}{2}\right) \alpha}+\sigma \Theta^{\bar{\sigma} \alpha}\left(M-\frac{\sigma}{2}\right) X^{\left(M-\frac{\sigma}{2}\right) \alpha ; M 2}\right] .
$$

We have a similar representation for operator of total spin $\boldsymbol{S}_{\mathrm{tot}}=\boldsymbol{S}+\frac{1}{2} \boldsymbol{\sigma}$ on a site [8]:

$$
\begin{aligned}
& S_{\mathrm{tot}}^{\eta}(i)=\sum_{M} \nu_{S}^{\eta}(M)\left[X_{i}^{(M+\eta) 0 ; M 0}+X_{i}^{(M+\eta) 2 ; M 2}\right]+\sum_{\alpha M^{\alpha}} \nu_{S+\frac{\alpha}{2}}^{\eta}\left(M^{\alpha}\right) X_{i}^{\left(M^{\alpha}+\eta\right) \alpha ; M^{\alpha} \alpha}, \\
& S_{\mathrm{tot}}^{z}(i)=\sum_{M} M\left[X_{i}^{M 0 ; M 0}+X_{i}^{M 2 ; M 2}\right]+\sum_{\alpha M^{\alpha}} M^{\alpha} X_{i}^{M^{\alpha} \alpha ; M^{\alpha} \alpha} .
\end{aligned}
$$

Here instead of two operators $S^{+}$and $S^{-}$we introduce one operator $S^{\eta}=1 / \sqrt{2}$. $\left(S^{x}+\mathrm{i} \eta S^{y}\right), \eta= \pm 1$. We also use a notation

$$
\nu_{S}^{\eta}(M)=\frac{1}{\sqrt{2}} \sqrt{(S-\eta M)(S+\eta M+1)} .
$$


One can see that the electron operator $c_{i \sigma}$ is presented by $X$-operators changing the number of electrons on a site by 1 . They are Fermi-like $X$-operators, obeying anticommutation permutative relations. The both spin operators $S_{i}^{\eta}$ and $S_{i}^{z}$ are presented by $X$-operators, changing electron number by 0 or 2 . They are considered to be Bose-like $X$-operators. The completeness condition should be fulfilled

$$
\sum_{M}\left(X_{i}^{M 0 ; M 0}+X_{i}^{M 2 ; M 2}\right)+\sum_{\alpha M^{\alpha}} X_{i}^{M^{\alpha} \alpha ; M^{\alpha} \alpha}=1 .
$$

One-site part $\mathcal{H}_{1}$ of the Hamiltonian (with magnetic field) can be written as

$$
\mathcal{H}_{1}=\sum_{i}\left[\sum_{M}\left(E_{M}^{0} X_{i}^{M 0 ; M 0}+E_{M}^{2} X_{i}^{M 2 ; M 2}\right)+\sum_{\alpha M^{\alpha}} E_{M^{\alpha}}^{\alpha} X_{i}^{M^{\alpha} \alpha ; M^{\alpha} \alpha}\right],
$$

where eigen-energies of site states are equal [7]

$$
\begin{aligned}
& E_{M}^{0}=-h M, \quad E_{M}^{2}=-h M-2 \mu, \\
& E_{M^{\alpha}}^{\alpha}=-h M^{\alpha}-\mu+E^{\alpha},
\end{aligned}
$$

( $E^{\alpha}$ is determined in (1.9), $h$ - magnetic field). In the expression for $\mathcal{H}_{1}$ we added a term $-\mu N$ with chemical potential.

To present a two-site part of the Hamiltonian $\mathcal{H}_{2}=\sum_{i j \sigma} t_{i j} c_{i \sigma}^{+} c_{j \sigma}$ it is worth introducing two-component spinors composed of Fermi-like $X$-operators:

$$
\Psi_{i}^{+}(\sigma \alpha M)=\left(X_{i}^{\left(M+\frac{\sigma}{2}\right) \alpha ; M 0}, \sigma X_{i}^{M 2 ;\left(M-\frac{\sigma}{2}\right) \alpha}\right)
$$

and column $\Psi_{i}$ composed of conjugated $X$-operators. Then $\mathcal{H}_{2}$ is written as

$$
\mathcal{H}_{2}=\sum_{i j} t_{i j} \sum_{\mathrm{I}_{1} \mathrm{I}_{2}} \Psi_{i}^{+}\left(\mathrm{I}_{1}\right) \mathbb{T}\left(\mathrm{I}_{1} \mathrm{I}_{2}\right) \Psi_{j}\left(\mathrm{I}_{2}\right)
$$

where

$$
\mathbb{T}\left(\mathrm{I}_{1} \mathrm{I}_{2}\right)=\delta_{\sigma_{1} \sigma_{2}}\left(\begin{array}{cc}
\Theta^{\sigma_{1} \alpha_{1}}\left(M_{1}+\frac{\sigma_{1}}{2}\right) \Theta^{\sigma_{2} \alpha_{2}}\left(M_{2}+\frac{\sigma_{2}}{2}\right) & \Theta^{\sigma_{1} \alpha_{1}}\left(M_{1}+\frac{\sigma_{1}}{2}\right) \Theta^{\bar{\sigma}_{2} \alpha_{2}}\left(M_{2}-\frac{\sigma_{2}}{2}\right) \\
\Theta^{\bar{\sigma}_{1} \alpha_{1}}\left(M_{1}-\frac{\sigma_{1}}{2}\right) \Theta^{\sigma_{2} \alpha_{2}}\left(M_{2}+\frac{\sigma_{2}}{2}\right) & \Theta^{\bar{\sigma}_{1} \alpha_{1}}\left(M_{1}-\frac{\sigma_{1}}{2}\right) \Theta^{\bar{\sigma}_{2} \alpha_{2}}\left(M_{2}-\frac{\sigma_{2}}{2}\right)
\end{array}\right) .
$$

Here we introduced a combined index $\mathrm{I}=(\sigma M \alpha \nu)$, where $\nu=1,2$ numerates the components of the spinor. Relations (2.8) and (2.12) present Hamiltonian of the sdmodel in terms of $X$-operators. The motion between two different sites is described by one-particle electron GF.

\section{Equation of motion for electron Green's function}

Let us introduce one-particle electron GF

$$
\mathcal{L}_{12}\left(\mathrm{I}_{1}, \mathrm{I}_{2}\right)=-\left(\begin{array}{cc}
\left\langle T \Psi_{1}\left(\mathrm{I}_{1}\right) \Psi_{2}^{+}\left(\mathrm{I}_{2}\right)\right\rangle_{V} & \left\langle T \Psi_{1}\left(\mathrm{I}_{1}\right) \Psi_{2}\left(\mathrm{I}_{2}\right)\right\rangle_{V} \\
\left\langle T \Psi_{1}^{+}\left(\mathrm{I}_{1}\right) \Psi_{2}^{+}\left(\mathrm{I}_{2}\right)\right\rangle_{V} & \left\langle T \Psi_{1}^{+}\left(\mathrm{I}_{1}\right) \Psi_{2}\left(\mathrm{I}_{2}\right)\right\rangle_{V}
\end{array}\right)
$$


where $\langle T \ldots\rangle_{V}$ means a statistical average of the system in the fluctuating fields $V$, that is

$$
\langle T \ldots\rangle_{V}=\frac{1}{Z[V]} \operatorname{Tr}\left(\mathrm{e}^{-\beta \mathcal{H}} T \ldots \mathrm{e}^{-V}\right) .
$$

Integer index is a combined one including a site number $i$ and time $\tau$, for example $1=\left(i_{1}, \tau_{1}\right)$.

We have to write the equation of motion for each matrix element in (3.1). For matrix element " 11 " one can use the identity

$$
\begin{aligned}
\frac{\partial}{\partial \tau_{1}}\left(\left(T \Psi_{1}\left(\mathrm{I}_{1}\right) \Psi_{2}^{+}\left(\mathrm{I}_{2}\right)\right)\right)= & \delta\left(\tau_{1}-\tau_{2}\right)\left(\left(T\left[\Psi_{1}\left(\mathrm{I}_{1}\right), \Psi_{2}^{+}\left(\mathrm{I}_{2}\right)\right]_{+}\right)\right) \\
& +\left(\left(T \dot{\Psi}_{1}\left(\mathrm{I}_{1}\right) \Psi_{2}^{+}\left(\mathrm{I}_{2}\right)\right)\right)-\left(\left(T\left[\Psi_{1}\left(\mathrm{I}_{1}\right), V\right]_{-} \Psi_{2}^{+}\left(\mathrm{I}_{2}\right)\right)\right) .
\end{aligned}
$$

Here we use a short notation:

$$
((T \ldots))=\operatorname{Tr}\left(\mathrm{e}^{-\beta \mathcal{H}} T \ldots \mathrm{e}^{-V}\right) .
$$

Now we must determine the operator $V$, describing the interaction with fluctuating fields. According to general conception of GFA, now we take $V$ in the form:

$$
\begin{aligned}
V= & v_{1^{\prime}}^{M_{1}^{\prime} 0 ; M_{1}^{\prime} 0} X_{1^{\prime}}^{M_{1}^{\prime} 0 ; M_{1}^{\prime} 0}+v_{1^{\prime}}^{M_{1}^{\prime} 2 ; M_{1}^{\prime} 2} X_{1^{\prime}}^{M_{1}^{\prime} 2 ; M_{1}^{\prime} 2} \\
& +v_{1^{\prime}}^{M_{1}^{\prime} 0 ; M_{1}^{\prime} 2} X_{1^{\prime}}^{M_{1}^{\prime} 2 ; M_{1}^{\prime} 0}+v_{1^{\prime}}^{M_{1}^{\prime} 2 ; M_{1}^{\prime} 0} X_{1^{\prime}}^{M_{1}^{\prime} 0 ; M_{1}^{\prime} 2} \\
& +v_{1^{\prime}}^{\left(M_{1}^{\prime}+\frac{\sigma_{2}^{\prime}}{2}\right) \alpha_{2}^{\prime} ;\left(M_{1}^{\prime}+\frac{\sigma_{1}^{\prime}}{2}\right) \alpha_{1}^{\prime}} X_{1^{\prime}}^{\left(M_{1}^{\prime}+\frac{\sigma_{1}^{\prime}}{2}\right) \alpha_{1}^{\prime} ;\left(M_{1}^{\prime}+\frac{\sigma_{2}^{\prime}}{2}\right) \alpha_{2}^{\prime}} .
\end{aligned}
$$

Here summation over all repeated primed indexes is implied.

As it is seen, we have to calculate first anticommutators of $\Psi$-operators. We have

$$
\left.\begin{array}{l}
\delta\left(\tau_{1}-\tau_{2}\right)\left[\Psi_{1}\left(\mathrm{I}_{1}\right), \Psi_{2}^{+}\left(\mathrm{I}_{2}\right)\right]_{+}=\delta_{12} \mathcal{F}\left(\mathrm{I}_{1}, \mathrm{I}_{2}\right) \\
\delta\left(\tau_{1}-\tau_{2}\right)\left[\Psi_{1}^{+}\left(\mathrm{I}_{1}\right), \Psi_{2}\left(\mathrm{I}_{2}\right)\right]_{+}=\delta_{12} \mathcal{F}^{+}\left(\mathrm{I}_{1}, \mathrm{I}_{2}\right) \\
\delta\left(\tau_{1}-\tau_{2}\right)\left[\Psi_{1}\left(\mathrm{I}_{1}\right), \Psi_{2}\left(\mathrm{I}_{2}\right)\right]_{+}=\delta_{12} \mathcal{Q}\left(\mathrm{I}_{1}, \mathrm{I}_{2}\right) \\
\delta\left(\tau_{1}-\tau_{2}\right)\left[\Psi_{1}^{+}\left(\mathrm{I}_{1}\right), \Psi_{2}^{+}\left(\mathrm{I}_{2}\right)\right]_{+}=\delta_{12} \mathcal{Q}^{+}\left(\mathrm{I}_{1}, \mathrm{I}_{2}\right)
\end{array}\right\} .
$$

Here $\mathcal{F}$ and $\mathcal{Q}$ are $2 \times 2$ matrices:

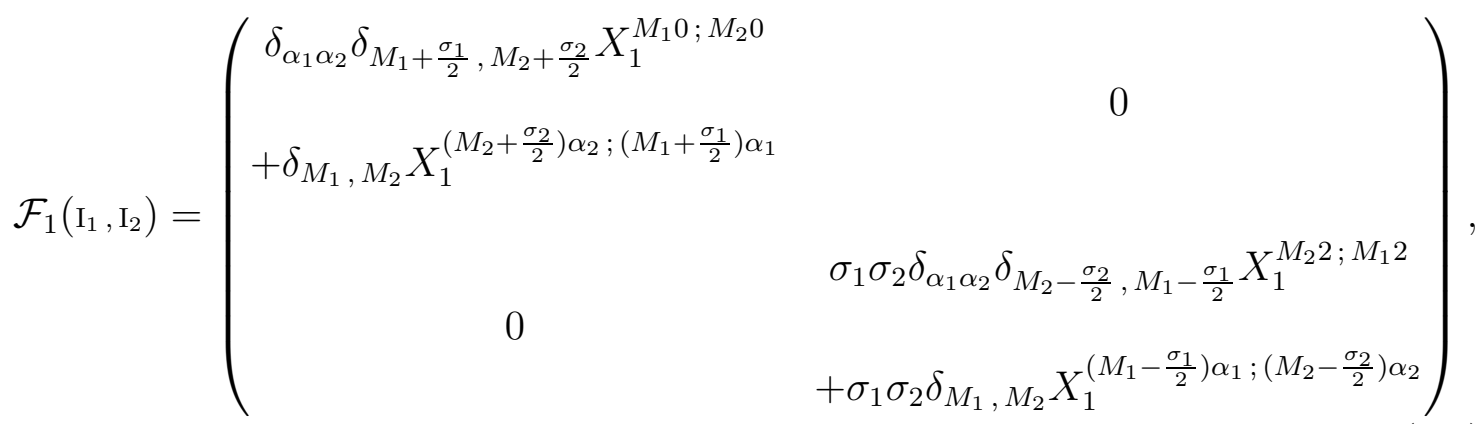

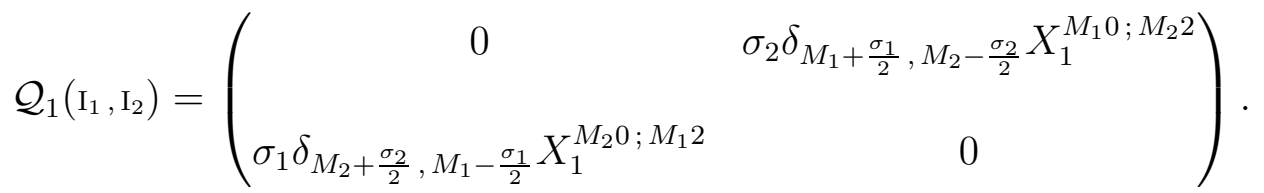


Matricies $\mathcal{F}^{+}$and $\mathcal{Q}^{+}$are Hermitian conjugated to matrices $\mathcal{F}$ and $\mathcal{Q}$.

We also need equations of motion for operators $\Psi$ and $\Psi^{+}$.

$$
\begin{aligned}
& \dot{\Psi}_{1}(\mathrm{I})=-\mathcal{E}\left(\mathrm{I}, \mathrm{I}^{\prime}\right) \Psi_{1}\left(\mathrm{I}^{\prime}\right)-\mathcal{F}_{1}\left(\mathrm{I}, \mathrm{I}^{\prime}\right) \mathcal{T}_{12^{\prime}}\left(\mathrm{I}^{\prime}, \mathrm{I}^{\prime \prime}\right) \Psi_{2^{\prime}}\left(\mathrm{I}^{\prime \prime}\right)+\mathcal{Q}_{1}\left(\mathrm{I}, \mathrm{I}^{\prime}\right) \mathcal{T}_{12^{\prime}}\left(\mathrm{I}^{\prime}, \mathrm{I}^{\prime \prime}\right) \Psi_{2^{\prime}}^{+}\left(\mathrm{I}^{\prime \prime}\right) \\
& \dot{\Psi}_{1}^{+}(\mathrm{I})=\mathcal{E}\left(\mathrm{I}, \mathrm{I}^{\prime}\right) \Psi_{1}^{+}\left(\mathrm{I}^{\prime}\right)-\mathcal{Q}_{1}^{+}\left(\mathrm{I}, \mathrm{I}^{\prime}\right) \mathcal{T}_{12^{\prime}}\left(\mathrm{I}^{\prime}, \mathrm{I}^{\prime \prime}\right) \Psi_{2^{\prime}}\left(\mathrm{I}^{\prime \prime}\right)+\mathcal{F}_{1}^{+}\left(\mathrm{I}, \mathrm{I}^{\prime}\right) \mathcal{T}_{12^{\prime}}\left(\mathrm{I}^{\prime}, \mathrm{I}^{\prime \prime}\right) \Psi_{2^{\prime}}^{+}\left(\mathrm{I}^{\prime \prime}\right)
\end{aligned}
$$

In this equations we use the notation

$$
\begin{aligned}
\mathcal{T}_{12}\left(\mathrm{I}_{1}, \mathrm{I}_{2}\right) & =\mathbb{T}\left(\mathrm{I}_{1}, \mathrm{I}_{2}\right) t_{i_{1} i_{2}} \delta\left(\tau_{1}-\tau_{2}\right) \\
\mathcal{E}\left(\mathrm{I}_{1}, \mathrm{I}_{2}\right) & =\left(\begin{array}{cc}
E^{\alpha}-\mu & 0 \\
0 & -E^{\alpha}-\mu
\end{array}\right) .
\end{aligned}
$$

Now we substitute the results (3.9), (3.11), (3.6) in equation (3.3) and in the similar equation for three other matrix elements of (3.1). As a result we come to the following equation for the matrix GF $\mathcal{L}$ :

$$
\left[\left(L_{0 V}^{-1}\right)_{11^{\prime}}\left(\mathrm{I}, \mathrm{I}^{\prime}\right)-(\widehat{A} \Phi Y)_{11^{\prime}}\left(\mathrm{I}, \mathrm{I}^{\prime}\right)-(\widehat{A} Y)_{11^{\prime}}\left(\mathrm{I}, \mathrm{I}^{\prime}\right)\right] \mathcal{L}_{1^{\prime} 2}\left(\mathrm{I}^{\prime}, \mathrm{I}^{\prime \prime}\right)=\left(\widehat{A}_{12} \Phi\right)\left(\mathrm{I}, \mathrm{I}^{\prime \prime}\right) .
$$

Here $\hat{A}$ is a matrix

$$
\widehat{A}_{12}\left(\mathrm{I}, \mathrm{I}^{\prime}\right)=\delta_{12}\left(\begin{array}{ll}
\widehat{F}_{1}\left(\mathrm{I}, \mathrm{I}^{\prime}\right) & \widehat{Q}_{1}\left(\mathrm{I}, \mathrm{I}^{\prime}\right) \\
\widehat{Q}_{1}^{+}\left(\mathrm{I}, \mathrm{I}^{\prime}\right) & \widehat{F}_{1}^{+}\left(\mathrm{I}, \mathrm{I}^{\prime}\right)
\end{array}\right)
$$

where quantities $\widehat{F}_{1}$ and $\widehat{Q}_{1}$ (and their conjugated ones) are given by matrixes (3.7) and (3.8), in which $X$-operators should be replaced by the corresponding functional derivatives according to a receipt

$$
X_{1}^{p q} \rightarrow-\frac{\delta}{\delta v_{1}^{q p}}
$$

Thus matrix $\widehat{A}$ is composed of the expressions, including functional derivatives. This is marked by cups over the letters.

Quantity $Y$ is the following $2 \times 2$ matrix

$$
Y_{12}\left(\mathrm{I}, \mathrm{I}^{\prime}\right)=\left(\begin{array}{cc}
\mathcal{T}_{12}\left(\mathrm{I}, \mathrm{I}^{\prime}\right) & 0 \\
0 & -\mathcal{T}_{12}\left(\mathrm{I}, \mathrm{I}^{\prime}\right)
\end{array}\right)
$$

Finally, the quantity $L_{0 V}^{-1}$ is also $2 \times 2$ matrix

$$
\left(L_{0 V}^{-1}\right)_{11^{\prime}}\left(\mathrm{I}, \mathrm{I}^{\prime}\right)=\left(\begin{array}{ll}
\left(G_{0 V}^{-1}\right)_{11^{\prime}}\left(\mathrm{I}, \mathrm{I}^{\prime}\right) & \delta_{11^{\prime}} W_{1}^{02}\left(\mathrm{I}, \mathrm{I}^{\prime}\right) \\
-\delta_{11^{\prime}} W_{1}^{20}\left(\mathrm{I}, \mathrm{I}^{\prime}\right) & \left(\widetilde{G}_{0 V}^{-1}\right)_{11^{\prime}}\left(\mathrm{I}, \mathrm{I}^{\prime}\right)
\end{array}\right) .
$$


Here

$$
\left(L_{0 V}^{-1}\right)_{11^{\prime}}\left(\mathrm{I}, \mathrm{I}^{\prime}\right)=-\left(\frac{\partial}{\partial \tau_{1}} \delta_{\mathrm{II}^{\prime}}+E_{1} \delta_{\mathrm{II} I^{\prime}}\right) \delta_{11^{\prime}}-\delta_{11^{\prime}} W_{1}\left(\mathrm{I}, \mathrm{I}^{\prime}\right)
$$

where

$$
\begin{aligned}
& W_{1}\left(\mathrm{I}_{1}, \mathrm{I}^{\prime}\right)=\delta_{M_{1}, M_{2}}\left(\begin{array}{cc}
-\delta_{\sigma_{1} \sigma_{2}} \delta_{\alpha_{1} \alpha_{2}} v_{1}^{M_{1} 0 ; M_{1} 0}+ & 0 \\
+v^{\left(M_{1}+\frac{\sigma_{2}}{2}\right) \alpha_{2} ;\left(M_{1}+\frac{\sigma_{1}}{2}\right) \alpha_{1}} & \delta_{\sigma_{1} \sigma_{2} \delta_{\alpha_{1} \alpha_{2}} v_{1}^{M_{1} 2 ; M_{1} 2}-} \\
0 & -v_{1}^{\left(M_{1}-\frac{\sigma_{1}}{2}\right) \alpha_{1} ;\left(M_{1}-\frac{\sigma_{2}}{2}\right) \alpha_{2}}
\end{array}\right), \\
& W_{1}^{02\left(\mathrm{I}_{1}, \mathrm{I}^{\prime}\right)}=\delta_{M_{1}, M_{2}} \delta_{\bar{\sigma}_{1} \sigma_{2}} \delta_{\alpha_{1} \alpha_{2}} v_{1}^{M_{1} 0 ; M_{1} 2}\left(\begin{array}{cc}
0 & 1 \\
-1 & 0
\end{array}\right) .
\end{aligned}
$$

So, all quantities in equation (3.13) have been determined. Remind that in this equation the summation over all repeated primed indexes is implied. Separate terms in the equation have the following physical meaning. The first term is a reversed GF of zero approximation (respectively hopping), but including the fluctuating fields. The second term determines Hartree-Fock correction due to hopping, and the third term includes the functional derivatives acting on the GF $\mathcal{L}$. In the right hand side there is $\widehat{A} \Phi$ quantity, which includes some averages of $X$-operators.

The form of equation (3.13) coincides with the form of equation for GF in the Hubbard model [9]. The only difference is in the form of matrices $\widehat{A}, Y$ and $L_{0 V}^{-1}$ as well as in the structure of the combined index I. The Hubbard model I includes spin $\sigma$ and spinor index $\nu$, while the sd-model $\mathrm{I}=(\sigma M \alpha \nu)$ includes two more indexes $M$ and $\alpha$.

We present an explicit form of matrix $\widehat{\mathcal{F}}$ and $\widehat{\mathcal{Q}}$, determining the operator matrix $\widehat{A}$. For simplicity we write them only in a particular but important case, when $\sigma_{1}=\sigma_{2} \equiv \sigma$.

$$
\begin{aligned}
& \widehat{\mathcal{F}}\left(\mathrm{I}_{1}, \mathrm{I}_{2}\right)=-\delta_{M_{1}, M_{2}}\left(\begin{array}{cc}
\delta_{\alpha_{1} \alpha_{2}} \frac{\delta}{\delta v_{1}^{M_{1} 0 ; M_{1} 0}} & 0 \\
+\frac{\delta}{\delta v^{\left(M_{1}+\frac{\sigma}{2}\right) \alpha_{2} ;\left(M_{1}+\frac{\sigma}{2}\right) \alpha_{1}}} & \delta_{\alpha_{1} \alpha_{2}} \frac{\delta}{\delta v_{1}^{M_{1} 2 ; M_{1} 2}} \\
0 & +\frac{\delta}{\delta v^{\left(M_{1}-\frac{\sigma}{2}\right) \alpha_{1} ;\left(M_{1}-\frac{\sigma}{2}\right) \alpha_{2}}}
\end{array}\right) \\
& \widehat{\mathcal{Q}}\left(\mathrm{I}_{1}, \mathrm{I}_{2}\right)=-\sigma\left(\begin{array}{cc}
0 & \delta_{M_{1}-M_{2}+\sigma, 0} \frac{\delta}{\delta v_{1}^{M_{1} 0 ;\left(M_{1}+\sigma\right) 2}} \\
\delta_{M_{1}-M_{2}-\sigma, 0} \frac{\delta}{\delta v_{1}^{\left(M_{1}-\sigma\right) 0 ; M_{1} 2}} & 0
\end{array}\right)
\end{aligned}
$$




\section{The Hubbard-I type approximation}

Consider a simple approximation, when in the basic equation (3.13) the term with functional derivatives of GF $\mathcal{L}$ is neglected. We rewrite the approximate equation with short notation

$$
\left[L_{0 V}^{-1}\left(\underline{1}^{\prime}\right)-(\hat{A} \Phi Y)\left(\underline{1}^{\prime}\right)\right] \mathcal{L}\left(\underline{1}^{\prime} \underline{2}\right)=(\hat{A} \Phi)(\underline{1} \underline{2}),
$$

where underlined indexes mean: $\underline{1}=\left(1 \mathrm{I}_{1}\right)$, etc. In a normal phase off-diagonal elements of matrix (3.1) vanish. Let us denote a diagonal element $\mathcal{L}^{11}=\mathcal{G}$, then from the matrix equation (4.1) one can write an equation for GF $\mathcal{G}$ :

$$
\mathcal{G}(\underline{1} \underline{2})=G\left(\underline{1} \underline{1}^{\prime}\right) \Lambda\left(\underline{1}^{\prime} \underline{2}\right)
$$

where $G(\underline{12})$ is a propagator part of $\mathcal{G}$, obeying the Dyson equation

$$
G^{-1}(\underline{1} \underline{2})=G_{0 V}^{-1}(\underline{1} \underline{2})-\Sigma(\underline{1} \underline{2})
$$

and the terminal part

$$
\Lambda(\underline{12})=(\widehat{A} \Phi)(\underline{1} \underline{2}) .
$$

The self-energy part in our approximation is equal to

$$
\Sigma(\underline{12})=(\widehat{A} \Phi Y)^{11}(\underline{12})=t_{12}\left(\widehat{\mathcal{F}}_{1} \mathbb{T} \Phi\right)\left(\mathrm{I}_{1}, \mathrm{I}_{2}\right) .
$$

Quantities $\widehat{\mathcal{F}}$ and $\mathbb{T}$ are determined by equation(3.21) and (2.13), respectively. The matrix, standing in equation(4.5) is factorized, which means that it can be written in the form

$$
\left(\widehat{\mathcal{F}}_{1} \mathbb{T} \Phi\right)\left(\mathrm{I}_{1}, \mathrm{I}_{2}\right)=\Lambda_{0}\left(\mathrm{I}_{1}\right) \theta\left(\mathrm{I}_{1}\right) \mathrm{T} \theta\left(\mathrm{I}_{2}\right) .
$$

Here $\mathrm{T}$ is a $2 \times 2$ matrix with all matrix elements equal to 1 .

$$
\begin{aligned}
& \Lambda_{0}(\mathrm{I})=\left(\begin{array}{cc}
\langle 0\rangle^{\sigma \alpha}(M) & 0 \\
0 & \langle 2\rangle^{\bar{\sigma} \alpha}(M)
\end{array}\right), \\
& \theta(\mathrm{I})=\left(\begin{array}{cc}
\theta^{\sigma \alpha}\left(M+\frac{\sigma}{2}\right) & 0 \\
0 & \theta^{\bar{\sigma} \alpha}\left(M-\frac{\sigma}{2}\right)
\end{array}\right) .
\end{aligned}
$$

Here we introduce the notation

$$
\left.\begin{array}{l}
\langle 0\rangle^{\sigma \alpha}(M)=\left\langle X^{M 0 ; M 0}\right\rangle+\left\langle X^{\left(M+\frac{\sigma}{2}\right) \alpha ;\left(M+\frac{\sigma}{2}\right) \alpha}\right\rangle \\
\langle 2\rangle^{\sigma \alpha}(M)=\left\langle X^{M 2 ; M 2}\right\rangle+\left\langle X^{\left(M-\frac{\sigma}{2}\right) \alpha ;\left(M-\frac{\sigma}{2}\right) \alpha}\right\rangle
\end{array}\right\} .
$$

The factorization (4.6) allows one to resolve the matrix equation (4.3). After Fourier transformation over the variable $(1-2)$ we obtain an equation for $G_{k}\left(\mathrm{I}_{1}, \mathrm{I}_{2}\right)$ and $\mathcal{G}_{k}\left(\mathrm{I}_{1}, \mathrm{I}_{2}\right)=G_{k}\left(\mathrm{I}_{1}, \mathrm{I}_{2}\right) \Lambda\left(\mathrm{I}_{2}\right)$. 
We present a solution of the obtained equation in the form:

$$
\mathcal{G}_{k}\left(\mathrm{I}_{1}, \mathrm{I}_{2}\right)=\mathcal{G}_{0 k}\left(\mathrm{I}_{1}\right) \delta_{\mathrm{I}_{1}, \mathrm{I}_{2}}+\mathcal{G}_{0 k}\left(\mathrm{I}_{1}\right) \theta\left(\mathrm{I}_{1}\right) \mathcal{D}(k) \mathrm{T} \theta\left(\mathrm{I}_{2}\right) \mathcal{G}_{0 k}\left(\mathrm{I}_{2}\right) .
$$

Here

$$
\begin{aligned}
\mathcal{G}_{0 k}(\mathrm{I}) & =\left(\begin{array}{cc}
\frac{\langle\theta\rangle^{\sigma \alpha}(M)}{\mathrm{i} \omega_{n}-E^{\alpha}+\mu} & 0 \\
0 & \frac{\langle 2\rangle^{\bar{\sigma} \alpha}(M)}{\mathrm{i} \omega_{n}+E^{\alpha}+\mu}
\end{array}\right), \\
\mathcal{D}(k) & =\left[1-\varepsilon_{\boldsymbol{k}} \sum_{\mathrm{I}} \mathrm{T} \theta(\mathrm{I}) \mathcal{G}_{0 k}(\mathrm{I}) \theta(\mathrm{I})\right]^{-1} .
\end{aligned}
$$

Notice that all quantities involved in equation (4.10) and equation (4.12) are $2 \times 2$ matrices.

For further analysis it is useful to determine a quantity

$$
\overline{\mathcal{G}}_{12}=\sum_{\mathrm{I}_{1} \mathrm{I}_{2}} \theta\left(\mathrm{I}_{1}\right) \mathcal{G}_{12}\left(\mathrm{I}_{1}, \mathrm{I}_{2}\right) \theta\left(\mathrm{I}_{2}\right)
$$

being a GF determined on electronic operators $c_{i}=\sum_{\sigma} c_{i \sigma}$, which are devided into two values: $c_{i}=g_{i}+h_{i}$, where $g_{i}$ includes only the first term in equation $(2.3)$, but $h_{i}$ - the second one, is connected with on-site transitions from double occupied states.

It is easy to derive the following expression for it

$$
\overline{\mathcal{G}}_{k}=\sum_{\mathrm{I}} \theta(\mathrm{I}) \mathcal{G}_{0 k}(\mathrm{I}) \theta(\mathrm{I}) \mathcal{D}(k) .
$$

After multiplying the matrices in the expressions (4.14) and (4.12) we obtain

$$
\begin{gathered}
\sum_{\mathrm{I}} \theta(\mathrm{I}) \mathcal{G}_{0 k}(\mathrm{I}) \theta(\mathrm{I})=\sum_{\mathrm{I}}\left(\begin{array}{cc}
\frac{\left(\theta^{2}\langle 0\rangle\right)(\mathrm{I})}{\mathrm{i} \omega_{n}-E^{\alpha}+\mu} & 0 \\
0 & \frac{\left(\bar{\theta}^{2}\langle 2\rangle\right)(\mathrm{I})}{\mathrm{i} \omega_{n}+E^{\alpha}+\mu}
\end{array}\right), \\
\mathcal{D}(k)=\frac{1}{d(k)}\left(\begin{array}{cc}
1-\varepsilon_{\boldsymbol{k}} \sum_{\mathrm{I}} \frac{\left(\bar{\theta}^{2}\langle 2\rangle\right)(\mathrm{I})}{\mathrm{i} \omega_{n}+E^{\alpha}+\mu} & \varepsilon_{\boldsymbol{k}} \sum_{\mathrm{I}} \frac{\left(\theta^{2}\langle 0\rangle\right)(\mathrm{I})}{\mathrm{i} \omega_{n}-E^{\alpha}+\mu} \\
\varepsilon_{\boldsymbol{k}} \sum_{\mathrm{I}} \frac{\left(\bar{\theta}^{2}\langle 2\rangle\right)(\mathrm{I})}{\mathrm{i} \omega_{n}+E^{\alpha}+\mu} & 1-\varepsilon_{\boldsymbol{k}} \sum_{\mathrm{I}} \frac{\left(\theta^{2}\langle 0\rangle\right)(\mathrm{I})}{\mathrm{i} \omega_{n}-E^{\alpha}+\mu}
\end{array}\right),
\end{gathered}
$$

where

$$
d(k)=1-\varepsilon_{\boldsymbol{k}} \sum_{\mathrm{I}}\left[\frac{\left(\theta^{2}\langle 0\rangle\right)(\mathrm{I})}{\mathrm{i} \omega_{n}-E^{\alpha}+\mu}+\frac{\left(\bar{\theta}^{2}\langle 2\rangle\right)(\mathrm{I})}{\mathrm{i} \omega_{n}+E^{\alpha}+\mu}\right] .
$$

Expressions (4.14)-(4.17) determine the matrix electron GF in the Hubbard-I approximation. All expressions include averaged values of diagonal $X$-operators determined by relations (4.9). In order to calculate them one has to know the electron $\mathrm{GF}$, constructed on $X$-operators for each expression (4.10). 
The poles of electron GF $\mathcal{G}_{k}\left(\mathrm{I}_{1}, \mathrm{I}_{2}\right)$ and $\overline{\mathcal{G}}_{k}$ are determined by zeroes of quantity $d(k)$. Since $\alpha$ takes two values, it is clear that dispersion relation $d(k)=0$ has four solutions unlike the Hubbard model, where two solutions exist, corresponding to lower and upper Hubbard subbands. The doubling of the solution numbers is connected with the fact that in sd-model, on each site there are two allowed states characterized by the value of total spin $j=S+1 / 2, j=S-1 / 2$.

\section{Limit of classical spin}

In the limit $S \rightarrow \infty\left|E^{+}\right|=\left|E^{-}\right|$, and the dispersion relation of the fourth order reduces to a square equation, determining two subbands $E_{1 \boldsymbol{k}}$ and $E_{2 \boldsymbol{k}}$. In paramagnetic phase the solution of equation $d(k)=0$ in the limit $S \rightarrow \infty$ gives two roots:

$$
E_{1,2 \boldsymbol{k}}=\varepsilon_{\boldsymbol{k}} \mp Q_{\boldsymbol{k}}, \quad Q_{\boldsymbol{k}}=\sqrt{\left(\frac{S J}{2}\right)^{2}+\varepsilon_{\boldsymbol{k}}^{2}} .
$$

Calculation using the equations (4.14)-(4.17) leads to the result for matrix elements of $\operatorname{GF} \overline{\mathcal{G}}(k) ;\left(\Omega=\mathrm{i} \omega_{n}+\mu\right)$ :

$$
\overline{\mathcal{G}}^{\mu \nu}(k)= \pm \mathcal{K}(\Omega)+\frac{\mathcal{P}_{1 \boldsymbol{k}}^{\mu \nu}}{\Omega-E_{1 \boldsymbol{k}}}+\frac{\mathcal{P}_{2 \boldsymbol{k}}^{\mu \nu}}{\Omega-E_{2 \boldsymbol{k}}},
$$

where

$$
\mathcal{K}(\Omega)=\langle 0\rangle\langle 2\rangle\left(\frac{1}{\Omega-\frac{S J}{2}}+\frac{1}{\Omega+\frac{S J}{2}}\right) .
$$

Sign "+" in (5.2) stands for diagonal matrix elements, and "-" stands for off diagonal ones, and

$$
\left.\begin{array}{l}
\mathcal{P}_{1,2 \boldsymbol{k}}^{11}=\mp\langle 0\rangle \frac{E_{1,2 \boldsymbol{k}}}{Q_{\boldsymbol{k}}} \pm\left(\frac{S J}{2}\right)^{2} \frac{\langle 0\rangle\langle 2\rangle}{Q_{\boldsymbol{k}} E_{1,2}} \pm 2\langle 0\rangle\langle 2\rangle \frac{\varepsilon_{\boldsymbol{k}}}{Q_{\boldsymbol{k}}} \\
\mathcal{P}_{1,2 \boldsymbol{k}}^{22}=\mp\langle 2\rangle \frac{E_{1,2 \boldsymbol{k}}}{Q_{\boldsymbol{k}}} \pm\left(\frac{S J}{2}\right)^{2} \frac{\langle 0\rangle\langle 2\rangle}{Q_{\boldsymbol{k}} E_{1,2 \boldsymbol{k}}} \pm 2\langle 0\rangle\langle 2\rangle \frac{\varepsilon_{\boldsymbol{k}}}{Q_{\boldsymbol{k}}} \\
\mathcal{P}_{1,2 \boldsymbol{k}}^{12}=\mathcal{P}_{1,2 \boldsymbol{k}}^{21}=\mp\left(\frac{S J}{2}\right)^{2} \frac{\langle 0\rangle\langle 2\rangle}{Q_{\boldsymbol{k}} E_{1,2 \boldsymbol{k}}} \mp 2\langle 0\rangle\langle 2\rangle \frac{\varepsilon_{\boldsymbol{k}}}{Q_{\boldsymbol{k}}}
\end{array}\right\}
$$

We have to know the electron GF, constructed on Fermi-operators

$$
G(k)=-\left\langle T c_{1} c_{2}^{+}\right\rangle_{k}=\sum_{\mu \nu} \overline{\mathcal{G}}^{\mu \nu}(k) .
$$

Substituting here the relations (5.4) and taking the equality $\langle 0\rangle+\langle 2\rangle=1$ (due to the completeness condition (2.7)) we find

$$
G(k)=\left(1-\frac{\varepsilon_{\boldsymbol{k}}}{Q_{\boldsymbol{k}}}\right) \frac{1}{\Omega-E_{1 \boldsymbol{k}}}+\left(1+\frac{\varepsilon_{\boldsymbol{k}}}{Q_{\boldsymbol{k}}}\right) \frac{1}{\Omega-E_{2 \boldsymbol{k}}} .
$$


It is remarkable that in the Hubbard-I type approximation statistical weights of the quasiparticle states do not depend on the electron concentration $n$; it enters only in the chemical potential $\mu$. However, at finite $S$ it should not be so, and it is necessary to solve the equation $d(k)=0$ of fourth order to define the quasiparticle energies.

An expression of the type (5.6) was obtained in [11] by decoupling the doubletime GFs in the first step. Obviously, the result (5.6) cannot give a metal-insulator phase transition because two subbands of quasiparticles with energies (5.1) are separated and should not be overlapped at any values of parameters. In the next step the authors [11] took into account the static contribution of spin fluctuations to the self-energy and show that it can lead to such a phase transition at some reasonable relations $J S \sim W$ between the parameters. However, there is some violation of sum rules for GFs, that demands a more precise approximation.

A final aim of our work is to study phase-transitions in the sd-model when dynamical fluctuations in the system are included. For this purpose the first order corrections over $W / S J$ in the terminal part $\Lambda_{1}$ and the second order in the self-energy $\Sigma_{2}$ over hopping will be calculated elsewhere. Similarly to what was done by us for the Hubbard model [9] we extract from $\Sigma_{2}$ a static contribution with some adjustive parameters, which are determined from fundamental conditions for electron GF [10]. Preliminary analysis shows that quasiparticle subbands can be overlapped which leads to a metal-insulator phase transition. Details of such a transition should be determined by interaction of quasiparticles with dynamical fluctuations. All these discussions will be a subject of next publication.

Authors thank Russian Foundation of Support of Science Schools, grant NS747.2003.2 and Division of Physical Sciences of the RAS, grant N 10104-71/OFN03/032-348/140705-126/01.06.2005. 


\title{
References
}

1. Izyumov Yu.A., Chaschin N.I., Yushankhai V.Yu., Phys. Rev. B, 2002, 65, 214425.

2. Izyumov Yu.A., Chaschin N.I., Phys. Met. Metallogr., 2002, 94, No. 6, 527; 2002, 94, No. 6, 539; 2004, 97, No. 3, 225.

3. Izyumov Yu.A., Alexeev D.S., Phys. Met. Metallogr., 2004, 97, No. 1, 5.

4. Kadanoff L.P., Baym G. Quantum Statistical Mechanics: Green's Function Methods in Equilibrium and Noneqvilibrium Problems. Benjamin, New York, 1962.

5. Izyumov Yu.A. - In: Lectures on the Physics of Highly Correlated Electron Systems VII, Seventh Training Course in the Physics of Correlated Electron Systems and HighTc Superconductors, ed. Avella A., Mancini F., AIP Conference Proceedings 678, 2003.

6. Izyumov Yu.A., Skryabin Yu.N. Basic Models in Quantum Theory of Magnetism. Ural Otd. Ros. Akad. Nauk, Ekaterinburg, 2002 (in Russian).

7. Erukhimov M.S., Ovchinnikov S.G., Phys. Stat. Sol. B, 1984, 123, 105.

8. Valkov V.V., Ovchinnikov S.G. Quasiparticles in Strongly Correlated Systems. Sib. Otd. Ros. Akad. Nauk, Novosibirsk, 2001 (in Russian).

9. Izyumov Yu.A., Chaschin N.I., Alexeev D.S., Manchini F., Eur. Phys. J. B, 2005, 45, 69.

10. Mancini F., Avella A., Adv. Phys., 2004, 53, No. 5-6, 537.

11. Anokhin A.O., Irkhin V.Yu., Katsnelson M.I., J. Phys.: Cond. Matt., 1991, 3, 1475.

\section{Підхід на основі генеруючого функціоналу до sd-моделі з сильними кореляціями}

\author{
Ю.А.Ізюмов, Н.І.Чащін, Д.С.Алєксєєв \\ Інститут фізики металів РАН, Уральське відділення \\ 620219 Єкатеринбург, Росія
}

Отримано 18 липня, 2005, в остаточному варіанті - 18 жовтня, 2005

Підхід генеруючого функціоналу типу Каданофа-Байма, розробленого раніше авторами для сильно скорельованих систем, застосовується до sd-моделі з сильною sd-взаємодією. Використовувався формалізм $X$-операторів Габбарда, і було отримано рівняння для електронних функцій Гріна з функціональними похідними по зовнішніх флуктуюючих полях. Ітерації в цьому рівнянні генерують теорію збурень біля атомної границі. В рамках цієї теорії розробляється наближення типу Хартрі-Фока, і обговорюється проблема фазового переходу метал-діелектрик в sd-моделі.

Ключові слова: теорії і моделі багатоелектронних систем, моделі граткового ферміону, сильно скорельовані електронні системи

PACS: $71.10 .-w, 71.10 . F d, 71.27 .+a$ 